\title{
High-throughput SNP genotyping in the highly heterozygous genome of Eucalyptus: assay success, polymorphism and transferability across species
}

Dario Grattapaglia ${ }^{1,2^{*}}$, Orzenil B Silva-Junior ${ }^{1}$, Matias Kirst ${ }^{3}$, Bruno Marco de Lima ${ }^{1,4}$, Danielle A Faria ${ }^{1}$ and Georgios J Pappas Jr ${ }^{1,2}$

\begin{abstract}
Background: High-throughput SNP genotyping has become an essential requirement for molecular breeding and population genomics studies in plant species. Large scale SNP developments have been reported for several mainstream crops. A growing interest now exists to expand the speed and resolution of genetic analysis to outbred species with highly heterozygous genomes. When nucleotide diversity is high, a refined diagnosis of the target SNP sequence context is needed to convert queried SNPs into high-quality genotypes using the Golden Gate Genotyping Technology (GGGT). This issue becomes exacerbated when attempting to transfer SNPs across species, a scarcely explored topic in plants, and likely to become significant for population genomics and inter specific breeding applications in less domesticated and less funded plant genera.
\end{abstract}

Results: We have successfully developed the first set of 768 SNPs assayed by the GGGT for the highly heterozygous genome of Eucalyptus from a mixed Sanger/454 database with 1,164,695 ESTs and the preliminary 4.5X draft genome sequence for E. grandis. A systematic assessment of in silico SNP filtering requirements showed that stringent constraints on the SNP surrounding sequences have a significant impact on SNP genotyping performance and polymorphism. SNP assay success was high for the 288 SNPs selected with more rigorous in silico constraints; $93 \%$ of them provided high quality genotype calls and $71 \%$ of them were polymorphic in a diverse panel of 96 individuals of five different species.

SNP reliability was high across nine Eucalyptus species belonging to three sections within subgenus Symphomyrtus and still satisfactory across species of two additional subgenera, although polymorphism declined as phylogenetic distance increased.

Conclusions: This study indicates that the GGGT performs well both within and across species of Eucalyptus notwithstanding its nucleotide diversity $\geq 2 \%$. The development of a much larger array of informative SNPs across multiple Eucalyptus species is feasible, although strongly dependent on having a representative and sufficiently deep collection of sequences from many individuals of each target species. A higher density SNP platform will be instrumental to undertake genome-wide phylogenetic and population genomics studies and to implement molecular breeding by Genomic Selection in Eucalyptus.

\footnotetext{
* Correspondence: dario@cenargen.embrapa.br

'EMBRAPA Genetic Resources and Biotechnology - Estação Parque Biológico,

final W5 norte, Brasilia, Brazil

Full list of author information is available at the end of the article
} 


\section{Background}

High-throughput, high density SNP genotyping has become an essential tool for QTL mapping, association genetics, gene discovery, germplasm characterization, molecular breeding and population genomics studies in several crops and model plants [1-7]. The abundance of Single Nucleotide Polymorphisms (SNPs) in plant genomes together with the rapidly falling costs and increased accessibility of genotyping technologies, have prompted an increasing interest to develop panels of SNP markers to expand resolution and throughput of genetic analysis in less-domesticated plant species with uncharacterized genomes such as those of orphan crops [8], forest [9-12] and fruit trees [13-15].

Two main strategies have been employed to identify SNPs in plants: utilization of EST sequence information to direct targeted amplicon resequencing and, more recently, next generation sequencing (NGS) technologies coupled or not to genome complexity reduction methods [16]. Amplicon resequencing of stretches of target genes is carried out in a germplasm panel that is relevant to the downstream applications and sufficiently large to avoid ascertainment bias. SNPs are mined in the resulting sequences and then assays are designed focusing on those particular SNPs. This strategy, although labor intensive, has been successful when the goal is to develop a moderate number of assayable SNPs [16]. High throughput NGS and direct in silico SNP identification now provide a very effective alternative to amplicon resequencing for SNP development in plants [17]. Thousands of SNPs can be readily identified given that sequences are obtained from an adequately large representation of individuals with sufficiently redundant genome coverage. Complexity reduction strategies such as using cDNA libraries $[18,19]$, AFLP derived representations [20], reduced representation libraries generated by restriction enzyme digestion and fragment selection [2,21], microarray-based [22] or in-solution [23] sequence capture, and additional target enrichment strategies [24] can be used to obtain the necessary sequence depth when the objective is to develop SNP based markers in specific genes or regions of the genome. Multiplexed bar-coded sequencing of such reduced genomic representations optimizes costs of SNP identification by increasing coverage and genotypic representation in the target regions [24-26]. Clearly the prospects are that sequence abundance and quality for SNP identification will no longer be a limiting factor for any plant genome.

A number of SNP genotyping technologies were developed in recent years mostly geared toward assaying human SNP variation. Among those that have been used in plant genetics, the Golden Gate Genotyping Technology (GGGT) developed by Illumina has consistently been reported as a reliable technology, displaying high levels of SNP conversion rate and reproducibility [16]. This assessment, initially reported for large scale human genotyping, has been corroborated in plant species including autogamous crops with low nucleotide diversity $(0.2 \%$ to $0.5 \%)$ [3,27-29] and outbred species with much higher sequence diversity typically $\geq 2 \%$ [9-13]. In highly heterozygous genomes, the development of GGGT SNP assays has been carried out mainly by amplicon resequencing targeting specific genes. This approach has been practical in conifers using haploid megagametophyte tissue $[30,31]$ and poplar for which a reference genome is available [12]. If attempted for large scale SNP development, however, this approach would be technically challenging for most outbred plant genomes due to the high levels of nucleotide diversity and additional indel variation as shown in earlier attempt for grape [32]. Direct SNP development from large in silico sequence resources will likely be the best approach for the highly heterozygous genomes of the majority of undomesticated plant species.

Irrespective of the method used to develop SNP markers in heterozygous genomes - direct in silico or targeted amplicon re-sequencing - challenges are faced in later steps when attempting to convert queried SNPs into high-quality genotypes. Particularly for the development of GGGT assays based on hybridization of allele and locus specific oligonucleotides, constraints have to be placed on the sequences flanking the target SNP [33]. A robust diagnosis of sequence variation in the vicinity of the target SNPs will depend largely on sequence coverage, sequence quality [34] and origin of sequences as far as the number and relatedness of individuals surveyed for SNP discovery. These issues will become increasingly exacerbated when attempting to transfer SNP assays across species within the same genus. Still a rarely explored topic in plants $[13,30,35]$, the assessment of inter-specific transferability of SNPs will likely be an important subject for population genomics and inter specific breeding applications in less domesticated and less funded plant genera.

Species of Eucalyptus are currently planted in more than 90 countries and are well known for their fast growth, straight form, valuable wood properties and wide adaptability [36]. Eucalyptus subgenus Symphyomyrtus, includes the majority of the twenty or so commercially planted species. E. globulus has been the top choice for plantations in temperate regions. Tropical Eucalyptus forestry, on the other hand, is based on interspecific hybrid breeding and clonal propagation with E. grandis as the pivotal species [36]. Molecular marker technologies have allowed a significant progress in the genetics and breeding of this vast genus that includes over 700 species [36]. Genetic analyses with molecular markers were key to settle phylogenetic issues 
[37], manage breeding populations [38] build linkage maps [39-41] and identify QTLs for important traits [42-45]. Nonetheless, more extensive genome coverage, higher throughput and improved inter specific transferability of current genotyping methods are necessary to increase resolution and speed for a variety of applications. A DArT array delivering around 3,000 to 5,000 dominant markers for mapping and population analyses was recently reported [46]. SNP developments in species of the genus have targeted specific candidate genes generating a few tens SNPs for specific association genetics studies $[47,48]$. However, large scale SNP arrays developments for Eucalyptus are yet to come. Due to their recent domestication, large population sizes and outbred mating system, species of Eucalyptus are among the ones with the highest frequency of SNPs reported in woody plant species and possibly in plants in general, with up to 1 SNP every 16 bp [49]. While a bonus for overall SNPs identification, such high nucleotide diversity, both within and among species, could represent an obstacle for the development of large sets of robust and polymorphic sets of Golden Gate assayable SNPs across species.

We are interested in developing genome-wide parallelized genotyping methods to be used for the operational implementation of Genomic Selection in Eucalyptus hybrid breeding, population genomics and phylogenetic studies in natural populations of the genus. The upcoming availability of a reference genome for Eucalyptus grandis and the rapid evolution of high throughput sequencing technologies will foster the buildup of large sequence dataset from many individuals, a valuable resource for the development of large collections of SNPs for the genus. In anticipation to this time, we used a 1.2 million mixed EST dataset including Sanger and 454 sequences from multiple Eucalyptus species and individuals to: (1) develop and validate an initial collection of genome-wide SNPs for Eucalyptus derived exclusively from in silico EST sequence data from unrelated individuals of different species; (2) assess the effect of increasingly stringent in silico SNP identification and design parameters on the reliability and polymorphism of SNP genotyping in species of Eucalyptus using the Golden Gate Genotyping Technology (GGGT); (3) evaluate SNPs transferability across eleven species of Eucalyptus and polymorphism in the five main planted species worldwide. Information on all SNPs discovered and validated in the present study is provided.

\section{Results}

EST clustering, contig assembly and SNP discovery pipeline

ESTs for six different species of Eucalyptus were used in this study to maximize the sampling of DNA sequence variation across species, although only a portion was retained for assembly after applying several quality filters. From a total of 136,041 Sanger-derived ESTs, 78,087 of them (57.4\%) were further processed. Similar percentage was retained out of the 1,028,654 454derived ESTs (60.7\%) (Table 1). The majority of the Sanger reads and all 454 reads were obtained from E. grandis, the pivotal species in most tropical breeding programs, totaling 94\% of the available ESTs before assembly and $96 \%$ after assembly, i.e. effectively used for SNP discovery. A two-step EST-assembly strategy was used: clustering performed at the species and sequencing technology levels followed by using the MIRA 2 assembler (Whole Genome Shotgun and EST Sequence Assembler) to consolidate the contigs and singletons from the previous step into a final EST assembly. After the MIRA assembly 48,973 contigs were obtained. Only those contigs formed by five or more ESTs were considered in this analysis to mitigate the limitations of alignment depth in SNP detection, thus resulting in 17,703 usable contigs (36.15\% of the total). From this contig set, SNPs were predicted using the program PolyBayes. Only SNPs with high probability $\left(P_{\mathrm{SNP}} \geq 0.99\right)$ were selected, totaling 162,141 potentially polymorphic sites (Figure 1).

\section{In silico selection of genome-wide SNP}

Five sequential filters were applied to the 162,141 candidate genome-wide SNPs for GGGT assay design from F0 (less stringent) to F4 (most stringent) (see Methods). When the filtering stringency increased from F0 to F4, the number of SNPs surviving selection in silico decreased abruptly. A total of 66,254 SNPs (40.6\%) were selected that had $\geq 5$ reads on the SNP position and a minimum of one read with the alternative base. This number dropped to 21,944 (13.5\%) when an in silico MAF $\geq 0.2$ constraint was applied and to $10,032(6.2 \%)$ when at least one EST from the more distant species $E$. globulus or E. gunnii was required in the contig. When the filter requiring flanking sequence conservation was applied, the number of SNPs selected dropped even

Table 1 Summary of the EST assembly for SNP discovery

\begin{tabular}{cccc}
\hline $\begin{array}{c}\text { Sequencing } \\
\text { technology }\end{array}$ & $\begin{array}{c}\text { Eucalyptus } \\
\text { species }\end{array}$ & $\begin{array}{c}\text { \# sequences used } \\
\text { for clustering }\end{array}$ & $\begin{array}{c}\text { \# sequences in } \\
\text { the assembly }\end{array}$ \\
\hline Sanger & E. grandis & 67,635 & 50,720 \\
& E. globulus & 30,260 & 10,088 \\
& E. urophylla & 7,755 & 4,387 \\
& E. gunnii & 19,586 & 7,018 \\
& E. pellita & 9,679 & 4,959 \\
454 & E. tereticornis & 1,126 & 1,095 \\
\hline TOTAL & E. grandis & $1,028,654$ & 623,922 \\
\hline
\end{tabular}




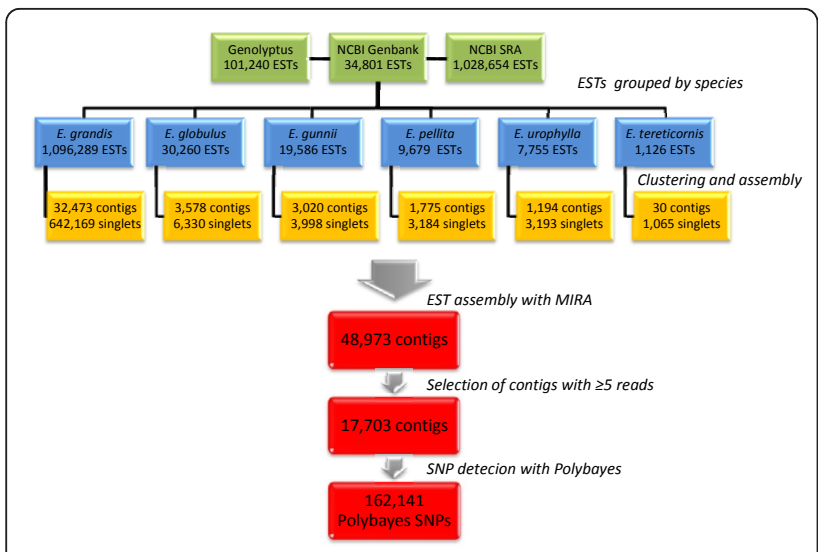

Figure 1 Flowchart with the output results of the EST clustering, contig assembly and SNP discovery pipeline prior to applying SNP filtering and selection for the GGGT assay design.

further to a final number of only 1,329 when a cutoff of 60 bases with no additional SNP on each side of the target SNP was stipulated. The number of unigene contigs retained along the filters also dropped significantly from an initial number of 17,703 to a mere 998 when all filtering constraints were applied (Table 2). Overall the proportion of SNPs with ADT (Assay design Tool) score greater than 0.6, i.e. SNPs with a high likelihood to be converted into a successful genotyping assay, was around 95\%, irrespective of the filtering treatments. For example, by applying only filter F0, 598 SNPs out of 621 had ADT score $\geq 0.6$; similarly, with filter F4, 525 out of 547 SNPs had ADT score $\geq 0.6$. The proportion of SNPs with ADT score $\geq 0.9$ was between 50 and $53 \%$ again showing no impact of the filtering treatments (Table 2). For bench validation only SNPs with ADT score $\geq 0.8$

Table 2 Summary of the in silico SNP development procedure using increasingly stringent SNP selection and design requirements (FO through F4) (see methods for details)

\begin{tabular}{|c|c|c|c|c|c|}
\hline $\begin{array}{l}\text { In silico SNP performance } \\
\text { assessment }\end{array}$ & Fo & F1 & F2 & F3 & F4 \\
\hline \# of SNPs & 66,254 & 21,944 & 10,032 & 3,187 & 1,329 \\
\hline \# of contigs with SNPs & 9,579 & 5,058 & 2,057 & 1,651 & 998 \\
\hline $\begin{array}{l}\text { \# of SNPs submitted to the } \\
\text { ADT }\end{array}$ & 621 & 605 & 583 & 367 & 547 \\
\hline $\begin{array}{l}\text { \# of SNPs with ADT Score } \geq \\
0.6\end{array}$ & 598 & 572 & 557 & 353 & 525 \\
\hline $\begin{array}{l}\% \text { of SNPs with ADT Score } \geq \\
0.6\end{array}$ & 96.3 & 94.5 & 95.5 & 96.2 & 96.0 \\
\hline $\begin{array}{l}\text { \# of SNPs with ADT Score } \geq \\
0.9\end{array}$ & 314 & 316 & 297 & 177 & 291 \\
\hline $\begin{array}{l}\% \text { of SNPs with ADT Score } \geq \\
0.9\end{array}$ & 50.6 & 52.2 & 50.9 & 48.2 & 53.2 \\
\hline \# of SNPs tested by the GGGT & 96 & 96 & 108 & 108 & 288 \\
\hline
\end{tabular}

were selected. A list of the 696 genome-wide SNPs selected and tested by the Golden Gate assay is available in Additional file 1.

\section{SNP discovery in pre-determined candidate genes}

From a list of 42 candidate genes selected from the literature as being putatively associated with relevant wood phenotypes in Eucalyptus (see Material and Methods), only in 20 of them SNPs were found that matched the minimum requirements of having $\geq 2$ reads with alternative bases at the SNP position and at least 60 bases of flanking sequence on each SNP side. For these 20 genes, a total of 175 SNPs were discovered and 72 were included in the bead array for downstream validation. These 72 SNPs were selected to assay at least one SNP in each one of the 20 genes and in those genes where several SNPs were available, SNPs that were derived from a contig with at least one read coming from E. globulus or E. gunnii and distantly positioned along the contig were selected. These 72 SNPs assayed in candidate genes are available as a separate spreadsheet in Additional file 1.

\section{SNP genotyping reliability}

The distributions of the proportions of SNPs in increasingly more reliable classes as measured by the GeneCall50 and GeneTrain scores for each in silico filter level were plotted (Figure 2). The relative distribution of the broken bars histograms corresponding to increasing levels of reliability suggests that when progressively more stringent in silico SNP selection requirements are applied from F0 to F4, larger proportions of SNPs with higher GeneTrain and GC50 scores were obtained. For SNPs in pre-determined candidate genes (CG) the proportions of SNPs at the lower ends of the distribution of GC50 and GeneTrain scores were larger reflecting the less stringent in silico selection applied in these cases (Figure 2). SNPs developed in specific candidate genes for which limitations existed regarding the number of available EST reads, generally showed a slightly lower performance in all measured parameters of reliability even when compared to SNPs developed only applying filter F0. The proportion of SNPs with call rate rate $\geq 95 \%$ was only $80.6 \%$, the average GeneTrain score was the lowest at 0.61 , and the proportion of SNPs with GeneTrain and GC50 scores $\geq 0.40$ was less than $90 \%$. However no difference was seen in the proportion of polymorphic SNPs in relation to the more stringent in silico filtering levels. Because SNPs in candidate genes were mined without observance of any specific in silico filtering level besides the most fundamental one (see methods), they were not included in the subsequent comparative analyses of the in silico filtering parameters. 

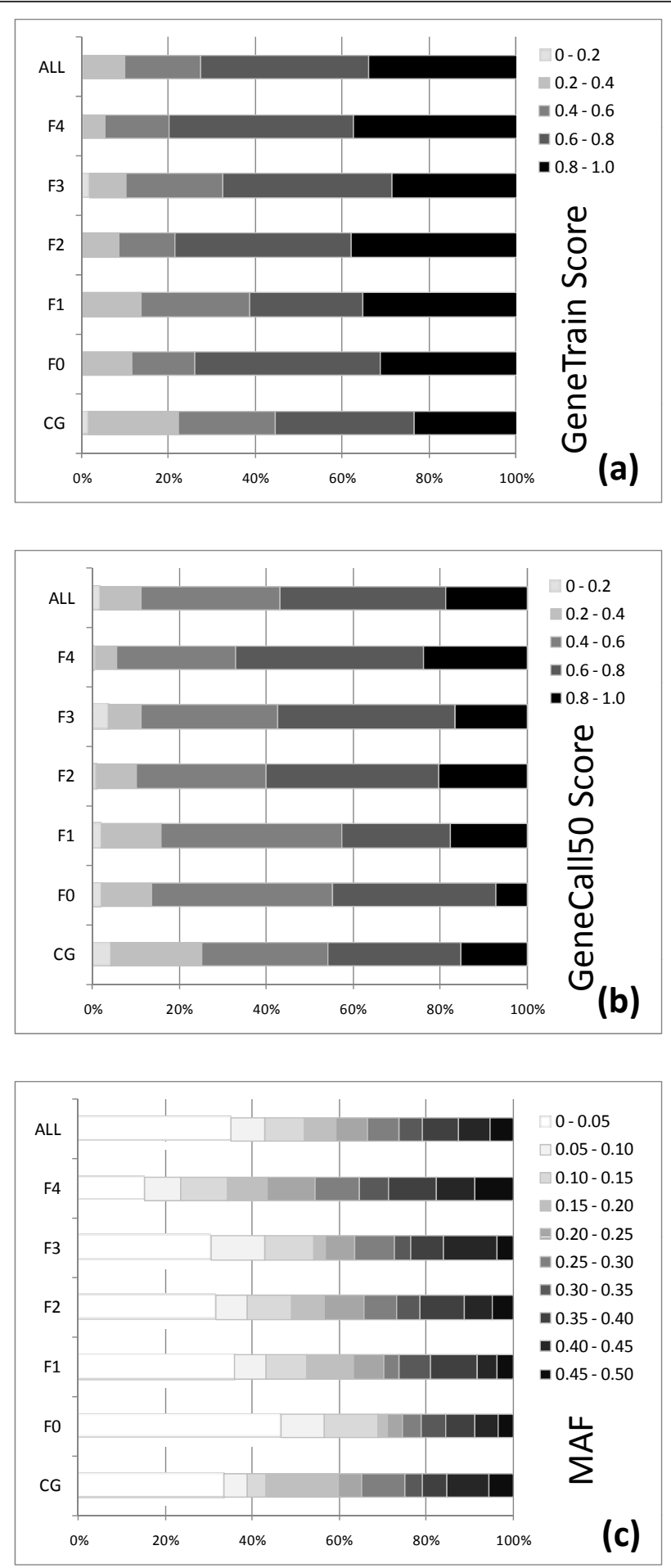

Figure 2 Distribution of the percentages of SNPs across classes of (a) GeneTrain Score; (b) GeneCall50 Score and (c) Minimum Allele Frequency (MAF) . Broken bars histograms are presented for all 768 SNPs together (ALL) and for each SNP category within the 696 genomewide SNPs selected by the different in silico filtering levels (F0 through F4 - see methods) and the 72 candidate gene (CG) SNPS. 
Table 3 Summary of the in vitro SNP genotyping performance assessed in a panel of 96 individuals from five Eucalyptus species

\begin{tabular}{|c|c|c|c|c|c|c|c|c|}
\hline In vitro SNP performance assessed & Candidate genes & Fo & F1 & F2 & F3 & $\mathrm{F} 4$ & Total counts & $\%$ \\
\hline \# SNPs tested by the GGGT & 72 & 96 & 96 & 108 & 108 & 288 & 768 & - \\
\hline Average SNP Call Rate (\%) & 91.0 & 95.2 & 90.0 & 94.9 & 95.0 & 97.8 & - & \\
\hline \# SNP with Call rate $\geq 0.95$ & 58 & 81 & 74 & 90 & 97 & 268 & 668 & 87.0 \\
\hline$\%$ SNP with Call rate $\geq 0.95$ & 80.6 & 84.4 & 77.1 & 83.3 & 89.8 & 93.1 & - & \\
\hline Average SNP GeneTrain score & 0.61 & 0.68 & 0.66 & 0.71 & 0.67 & 0.72 & - & \\
\hline \# SNPs with GeneTrain score $\geq 0.40$ & 64 & 90 & 90 & 100 & 101 & 278 & 723 & 94.1 \\
\hline$\%$ SNPs with GeneTrain score $\geq 0.40$ & 88.9 & 93.8 & 93.8 & 92.6 & 93.5 & 96.5 & - & \\
\hline Average SNP GC50 score & 0.57 & 0.59 & 0.59 & 0.64 & 0.62 & 0.67 & - & \\
\hline \# SNPs with GC50 score $\geq 0.40$ & 63 & 89 & 89 & 100 & 101 & 277 & 719 & 93.6 \\
\hline$\%$ SNPs with GC50 score $\geq 0.40$ & 87.5 & 92.7 & 92.7 & 92.6 & 93.5 & 96.2 & - & \\
\hline Average MAF of SNPs with MAF $\geq 0.05$ & 0.26 & 0.24 & 0.25 & 0.26 & 0.25 & 0.27 & - & \\
\hline \# SNP with MAF > 0.05 & 51 & 48 & 55 & 75 & 74 & 205 & 508 & 66.1 \\
\hline$\%$ SNP with MAF > 0.05 & 70.8 & 50.0 & 57.3 & 69.4 & 68.5 & 71.2 & - & \\
\hline
\end{tabular}

Averages and SNP counts above specific thresholds of SNP reliability parameters (Call Rate, GeneCall50, GeneTrain scores) and polymorphism (MAF) for SNPs in pre-selected candidate genes and for genome-wide SNPs selected with increasingly stringent in silico SNP selection and design requirements (F0 through F4 see methods for details).

The overall genotyping reliability for the 768 SNPs was assessed by estimating SNP counts above conventionally used threshold and average values for Call Rate, GeneCall and GeneTrain scores (Table 3). Goodness-offit for normality tests showed that all these three variables were not normally distributed $(\mathrm{p}<0.0001)$. The average call rates for all SNPs, irrespective of in silico filter levels were above 90\%; $87 \%$ of all 768 SNPs had call rates $\geq 95 \%$. Mann-Whitney non-parametric tests showed no significant difference in average call rate and GeneTrain score between filtering levels tested individually or combined based on requirements of conservation of flanking sequences $(\mathrm{F} 0+\mathrm{F} 1+\mathrm{F} 2$ against $\mathrm{F} 3+\mathrm{F} 4)$. The proportion of SNPs with call rates $\geq 95 \%$ varied, with an increasing trend when going toward a more stringent SNP filtering selection and reaching 93.1\% with filter F4. When tested pair-wise and sequentially, i.e. F0 against F1, F1 against F2 and so on, no significant differences in the proportion of SNPs with call rates $\geq 95 \%$ or GeneTrain $\geq 0.4$ were found using a Chi-square Pearson test. However when the pooled count of all SNPs selected with no requirements of conservation of flanking sequences (filters F0+F1+F2; 245 in 300) was compared to the count of SNPs selected with such requirements (i. e. no additional SNPs either in 20 or 60 bases on each SNP side, i.e. filters F3+F4; 365 SNPs in 396) (Table 3), a highly significant difference was found in the final number of SNPs recovered with call rates $\geq 95 \%$, (Chisquare Pearson $=17.40 ; \mathrm{p}=0.00003)$. SNP reliability based on the GeneCall50 score followed a similar trend observed with the Call Rate and GeneTrain with an increase from 0.59 for F0 to 0.67 for F4. However a significant difference in the average GC50 score was found when the comparison was between the pooled SNPs from filters $\mathrm{F} 0+\mathrm{F} 1+\mathrm{F} 2(\mathrm{GC} 50=0.61)$ and those derived from filters $\mathrm{F} 3+\mathrm{F} 4(\mathrm{GC50}=0.66)$ (Mann-Whitney nonparametric test $\mathrm{p}=0.000041)$. These results indicate that although the vast majority of SNPs could be robustly scored with high call rate, a more stringent in silico selection on the flanking sequences yields more SNPs with higher call rates and GeneTrain scores as well as SNPs with average higher GeneCall50 scores. We used a relatively stringent GeneCall50 cutoff of 0.4 when compared to other SNP development studies as we observed that at lower thresholds, the genotype cluster separation consistently showed undesirable shifts.

\section{SNP polymorphism}

The proportion of polymorphic SNPs overall the five main Eucalyptus species ( $\mathrm{N}=96$ individuals) for all 768 SNPs was estimated at $66.1 \%$, which corresponds to the conversion rate. When only the 711 SNPs that simultaneously met the adhoc thresholds of reliability (GC50 $\geq$ 0.4 and Call Rate $\geq 95 \%$ ) are considered, a higher proportion of them are polymorphic with MAF $\geq 0.05$ (505 in 711) i.e. a conversion rate of $71 \%$. The average MAF of polymorphic SNPs was consistently around 0.25 for all filtering levels and for the candidate gene SNPs as well (Table 3). The proportion of SNPs with higher polymorphism level, measured by MAF, increased as progressively more stringent selection was applied in silico as depicted in the broken bars histogram. However only with the more rigorous F4 selection on the SNP flanking sequence a larger proportion of polymorphic SNPs was effectively recovered (Figure 2). No increase was seen in the proportion of polymorphic SNPs when going from filter F1 $(69.4 \%)$ to F2 (68.5\%), i.e. by including the requirement of ESTs reads from section 
Maidenaria in the contig (Table 3). However the proportion of polymorphic SNPs significantly increased from selection with filters F0+F1+F2 (175 in 300) to selection with filters F3+F4 (279 in 396) (Chi-square Pearson = 9.36; $\mathrm{p}=0.00221$ ), suggesting that the inclusion of a filtering requirement on the SNP flanking sequences not only results in more reliably assayable SNPs but also increases the proportion of polymorphic SNPs.

The proportions of polymorphic SNPs were also estimated for each main species separately, and for all possible combinations of species, i.e. the number of SNPs that were polymorphic for the species simultaneously (Table 4 and Additional file 2). In this analysis only the 711 SNPs that simultaneously met the adhoc thresholds of reliability were considered. The highest proportions of polymorphic SNPs were observed for E. grandis, E. urophylla and E. camaldulensis, between $40.9 \%$ and $49.4 \%$, while in the two species of the more distant section Maidenaria, the proportion of polymorphic SNPs was around 22 to $25 \%$. The average number of polymorphic SNPs in all three-way species combinations varied from a maximum of 144 (20\%) for the E. grandis, $E$. urophylla and E. camaldulensis set to a minimum of 77 (11\%) for the E. urophylla, E. globulus and E. camaldulensis set. Only between 64 and 78 SNPs were polymorphic when any four species combinations were considered and only 55 (7.7\%) when all five were taken into account (Additional file 2). Given the relatively limited sample size, when a less conservative estimate of polymorphism within species was used (MAF $\geq 0.01$ ) the proportions of polymorphic SNPs increased considerably in all species and combinations. For example in $E$. grandis the proportion went from $49.4 \%$ to $62 \%$, in $E$. camaldulensis from $41.2 \%$ to $58.5 \%$ and in E. globulus from $22.2 \%$ to $33.6 \%$. Likewise SNPs that were polymorphic in two or more species concurrently also increased.

\section{SNP reliability across subgenera}

Based on the results showing a significant increase in SNP genotyping reliability when introducing in silico constraints on SNP flanking sequences, SNP reliability across a larger set of species and subgenera was evaluated by considering only two overall SNP selection levels: (1) SNPs selected with no requirement of conservation of flanking sequences (this group includes candidate genes SNPs plus genome-wide SNPs from filters F0 $+\mathrm{F} 1+\mathrm{F} 2$, totaling $372 \mathrm{SNPs}$ ) and (2) SNPs selected requiring conservation in flanking sequences of either 20 or 60 bases (this group includes genome-wide SNPs from filters F3+F4 with a total of 396 SNPs). Reliability was assessed by the counts and proportion of SNPs that displayed a Call rate $\geq 0.95$ and a GC50 score $\geq 0.40$ (Table 5). A comparison of the GeneTrain score across species does not apply in this case, as it is a SNP specific statistics appraising the quality of the genotype clusters and remains unchanged for all samples used to generate the clusters. The relative proportions of reliable SNPs across all nine species of subgenus Symphyomyrtus did not vary much within each SNP selection level. With no flanking sequence constraints on average $81 \%$ of the SNPs had call rate $\geq 0.95$ and $88 \%$ a GC50 score $\geq 0.40$. With flanking sequence constraints the proportions were higher, $90 \%$ of the SNPs had call rate $\geq 0.95$ and $94 \%$ a GC50 score $\geq 0.40$. However, a lower genotyping reliability was observed for the two species outside subgenus Symphyomyrtus, with only around 50\% of the SNPs having satisfactory call rate and GC50 scores even for SNPs selected with flanking sequence constraints. In all eleven species but E. cloeziana, a significant increase was found (Pearson chi square test $p$ $<0.01)$ in the number of SNPs that met or exceeded the call rate and GC50 thresholds when flanking sequence constraints were applied in silico (Table 5). This result confirms the impact of flanking sequence constraints on the reliability of SNPs in all tested species, irrespective of the presence of ESTs from the particular species in the database used for SNP discovery.

\section{Heritability-based SNP validation}

SNP assay quality was further assessed by estimating heritability of allelic transmission in parent-parent-offspring trios involving different Eucalyptus species as parents. Heritability is defined as the number of offspring genotypes that agree with the expected inheritance over the total number of genotype calls possible. In family E. grandis $\times E$. urophylla $(\mathrm{G} \times \mathrm{U})$ there were 457 Mendelian transmission inconsistencies out of the

Table 4 Counts and percentages of polymorphic SNPs (MAF $\geq 0.05$ ) from a total of 711 reliable SNPs, in each one of the five main Eucalyptus species surveyed (diagonal) and in pair-wise sets of species (above the diagonal)

\begin{tabular}{|c|c|c|c|c|c|}
\hline & E. grandis & E. urophylla & E. globulus & E. nitens & E. camaldulensis \\
\hline E. grandis & 351 (49.4\%) & 209 (29.4\%) & 117 (16.5\%) & $128(18.0 \%)$ & $194(27.3 \%)$ \\
\hline E. urophylla & & 291 (40.9\%) & 107 (15.0\%) & 120 (16.9\%) & 187 (26.3\%) \\
\hline E. globulus & & & $158(22.2 \%)$ & 104 (14.6\%) & $118(16.6 \%)$ \\
\hline E. nitens & & & & 181 (25.5\%) & 127 (17.9\%) \\
\hline E. camaldulensis & & & & & 293 (41.2\%) \\
\hline
\end{tabular}


Table 5 Summary of SNP reliability across species, sections and subgenera of Eucalyptus as measured by the number of SNP meeting the thresholds of call rate and GeneCall50 for two groups of SNPs that differed regarding the flanking sequence constraints during in silico SNP mining and GGGT assay design

\begin{tabular}{|c|c|c|c|c|c|c|c|c|c|}
\hline \multirow[b]{2}{*}{ Subgenera/Section } & \multirow[b]{2}{*}{ Species } & \multicolumn{4}{|c|}{$\begin{array}{l}\text { SNPs selected with no flanking sequence } \\
\text { requirements }(\mathrm{N}=372)\end{array}$} & \multicolumn{4}{|c|}{$\begin{array}{l}\text { SNPs selected with no additional SNPs in } \\
\text { flanking sequence }(N=396)\end{array}$} \\
\hline & & $\begin{array}{c}\text { \# SNPs } \\
\text { with } \\
\text { Call rate } \\
\geq 95 \%\end{array}$ & $\begin{array}{l}\% \text { SNPs } \\
\text { with } \\
\text { Call rate } \\
\geq 95 \%\end{array}$ & $\begin{array}{c}\text { \# SNPs } \\
\text { with } \\
\text { GC50 } \\
\geq 0.40\end{array}$ & $\begin{array}{c}\% \text { SNPs } \\
\text { with } \\
\text { GC50 } \\
\geq 0.40\end{array}$ & $\begin{array}{c}\text { \# SNPs } \\
\text { with } \\
\text { Call rate } \\
\geq 95 \%\end{array}$ & $\begin{array}{l}\% \text { SNPs } \\
\text { with } \\
\text { Call rate } \\
\geq 95 \%\end{array}$ & $\begin{array}{c}\text { \# SNPs } \\
\text { with } \\
\text { GC50 } \\
\geq 0.40\end{array}$ & $\begin{array}{c}\% \text { SNPs } \\
\text { with } \\
\text { GC50 } \\
\geq 0.40\end{array}$ \\
\hline Symphyomyrtus/Latoangulatae & E. grandis & 323 & 86.8 & 333 & 89.5 & 378 & 95.5 & 378 & 95.5 \\
\hline Symphyomyrtus/Latoangulatae & E. urophylla & 310 & 83.3 & 335 & 90.1 & 369 & 93.2 & 377 & 95.2 \\
\hline Symphyomyrtus/Latoangulatae & E. saligna & 279 & 75.0 & 328 & 88.2 & 343 & 86.6 & 376 & 94.9 \\
\hline Symphyomyrtus/Maidenaria & E. globulus & 325 & 87.4 & 331 & 89.0 & 369 & 93.2 & 374 & 94.4 \\
\hline Symphyomyrtus/Maidenaria & E. nitens & 311 & 83.6 & 327 & 87.9 & 369 & 93.2 & 375 & 94.7 \\
\hline Symphyomyrtus/Maidenaria & E. dunnii & 295 & 79.3 & 324 & 87.1 & 361 & 91.2 & 371 & 93.7 \\
\hline Symphyomyrtus/Maidenaria & E. viminalis & 300 & 80.6 & 325 & 87.4 & 353 & 89.1 & 370 & 93.4 \\
\hline Symphyomyrtus/Exsertaria & E. camaldulensis & 289 & 77.7 & 336 & 90.3 & 339 & 85.6 & 376 & 94.9 \\
\hline Symphyomyrtus/Exsertaria & E. tereticornis & 281 & 75.5 & 319 & 85.8 & 330 & 83.3 & 365 & 92.2 \\
\hline Eucalyptus/Pseudophloius & E. pilularis & 194 & 52.2 & 271 & 72.8 & 246 & 62.1 & 325 & 82.1 \\
\hline Idiogenes/Gympiaria & E. cloeziana & 166 & 44.6 & 223 & 59.9 & 198 & 50.0 & 278 & 70.2 \\
\hline
\end{tabular}

36,864 allelic transmissions assayed, i.e. a genotyping miscall rate of $1.2 \%$. In total 719 SNPs out of the 768 tested $(93.6 \%)$ had $100 \%$ heritability and $80 \%$ of the inheritance miscalls were concentrated in 24 SNPs. In the four species family $([E$. dunni $\times$ E. grandis $] \times[E$. urophylla $\times$ E. globulus]) $(\mathrm{DG} \times \mathrm{UGL})$ 1,596 transmission inconsistencies were seen, i.e. a genotyping miscall rate of $4.3 \%$, only 678 SNPs $(88.3 \%)$ had $100 \%$ heritability and $80 \%$ of the inheritance miscalls were concentrated in 71 SNPs. Only 17 SNPs displayed miscalls in both families concurrently, revealing potentially more problematic SNPs. Upon inspection of the SNPs clustering graphs most inheritance miscalls in both families were due to the two parents being homozygous AA and $\mathrm{BB}$ and offspring not having the expected genotype $\mathrm{AB}$ but rather one of the two homozygous ones.

\section{Sequence-based validation of SNP genotypes}

SNP validation was possible for 50 SNPs for which five or more genomic reads overlapping at the SNP position with sequence quality $\mathrm{Q} \geq 20$ were obtained. Given the limited sample size available (number of observed reads at the SNP site) a less conservative alpha level $\alpha=0.1$ was used to increase the power of the binomial test used to declare sequence-based genotypes. In other words, by increasing the chance of obtaining a statistically significant result, the probability of correctly declaring a sequence-based homozygous genotype in spite of the small number of observed reads was increased although at the expense of an increase in Type I error, i.e. erroneously declaring the genotype as homozygous when in fact it is heterozygous. Sequencebased genotypes at 43 of the 50 SNPs (86\%) matched the Golden Gate assay called genotypes (Additional file $3)$.

\section{Discussion}

We have successfully developed the first set of 768 SNPs assayed by the Golden Gate genotyping technology for the highly heterozygous genome of Eucalyptus. The overall SNP success rate was high, with $87 \%$ of all SNPs showing call rates $\geq 95 \%, 94.1 \%$ of them having a GeneTrain score $\geq 0.40$ and $93.6 \%$ a GeneCall50 score $\geq$ 0.40 . The conversion rate, which is the proportion of polymorphic SNPs divided by the total number of SNPs was $66.1 \%$ estimated in a diverse panel of 96 individuals of five different species (Table 3). These are the first results of a larger scale SNP development effort for Eucalyptus suggesting that the Golden Gate assay performs well both within and across species notwithstanding the high nucleotide diversity of the complex Eucalyptus genome and the wide range of species for which SNP genotyping is pursued.

\section{SNP discovery and selection from Eucalyptus ESTs}

SNP discovery and assay development was carried out based on all available 1,164,695 ESTs in public and our own databases as of May 2009 (Table 1). Although this was considered a large EST set by pre-next-generation sequencing standards, it constitutes a relatively small collection given today's sequencing technologies. A large number $(162,141)$ of potentially polymorphic sites was found after EST clustering and assembly in agreement with the previous abundance of SNPs reported for species of Eucalyptus from in silico surveys [18,49]. However only $36 \%$ of the assembled contigs met the depth 
requirement of five reads overlapping the SNP position with 60 bases of available sequence on each side recommended for Golden Gate genotyping (Figure 1). In fact when SNPs were searched in 42 pre-determined candidate genes of interest, only 20 of them were available for SNP assay design. This result suggests that if SNPs are to be developed for specific genes from direct in silico sequence resources, a much higher sequence coverage than the one used in this work is necessary. Recently, such an approach proved successful by massive sequencing of reduced representation libraries of multiple grape varieties to develop a $\sim 9,000$ selected SNP array from over 470,000 in silico detected SNPs [13]. Several genetically heterogeneous plant genomes should be amenable to this same SNP development approach opening concrete perspectives for high throughput genotyping in a large number of less characterized, largely undomesticated species.

\section{SNP reliability is enhanced by stringent in silico constraints}

Knowledge of the SNP flanking sequences is an important aspect of the success of the Golden Gate assay. The assay design tool provided by Illumina checks for the presence of repetitive or palindromic sequences, GC content and neighboring polymorphisms to provide a functionality score for each candidate SNP [33]. However no systematic assessment of the impact of additional polymorphisms in the flanking sequence of the target SNP on its genotyping reliability has been reported. While this represents a minor concern for species of low nucleotide diversity such as humans, crop plants and domestic animals, it is a key issue for highly heterozygous genomes with nucleotide diversity in excess of $1 \%$. In the heterogeneous genome of loblolly pine, for example, Eckert et al. [9] suggested that the SNP success rate observed (67\%), lower than the typical $\geq 90 \%$ rate obtained in crop plants and humans, could be attributed to the presence of undetected SNPs in the flanking sequences, but no detailed assessment of this issue was carried out. In spruce, no specific selection for conserved flanking sequences was carried out during SNP development; SNP success rates were around 69 to $77 \%$ [11]. In Pinus pinaster, the proportion of successful SNPs (GeneTrain > 0.25) developed from in silico was estimated at $61.5 \%$ while for SNPs developed by targeted amplicon resequencing it was slightly higher, at $73 \%$ but also no specific selection for more conserved SNP flanking sequences was carried out [10].

In our study we used five sequential in silico filters on the initial set of 162,141 candidate genome-wide SNPs discovered in 17,703 EST contigs that had $\geq 5$ reads. While filter F0 was a commonly used criterion for SNP discovery in silico, F1 added a requirement for a minimum in silico estimated MAF $\geq 0.2$. This single additional requirement, however, reduced to less than $1 /$ 3 the number of available SNPs for assay design (Table 2 ). Filter F2 introduced a requirement of inter-specific sequence representation in the contig to increase sequence sampling both at the SNP position as well as for flanking sequences, in an attempt to increase SNP transferability across more distant species. This further filter caused a reduction of $50 \%$ in the number of available SNPs. When filters F3 and F4 added a progressively more rigorous requirement on the SNP flanking sequences, the number of surviving SNPs decreased rapidly to a point that only 3,187 SNPs in 1,651 genes remained for SNP assay design after filter F3 or 1,329 SNPs in 998 genes after F4 (Table 2). The application of similarly stringent in silico quality filters to the initial SNP source also caused a 10-fold reduction in the available putative SNP when developing a 54,000 SNP array for bovine, but resulted in an increase from $50 \%$ to $>85 \%$ in the conversion rate [50]. In our study, however, it is important to note that the observed reduction in the number of available SNPs was largely a result of the relatively limited number of ESTs available at the beginning of the pipeline $(702,009)$, many derived from short 454 reads, so that the minimum in silico MAF $\geq 0.2$ and sufficient flanking sequences could not be achieved in most contigs. Additionally only $\sim 17,000$ ESTs from section Maidenaria (E. globulus plus E. gunnii) were available among the 702,009 used (only 2.4\%), strongly limiting the ability to fulfill the requirement of filter F2. This highly unbalanced sequence representation most likely was responsible for this sharp decrease in sequences used for SNP assay design. Had we had access to a more balanced EST representation across species, a much larger number of SNPs would probably have survived all sequential filters and be amenable to assay design.

Our results show that the increasingly more stringent requirements on the SNP surrounding sequences are highly effective and have a statistically significant impact not only on SNP reliability but also on the proportion of polymorphic SNPs. Significantly more SNPs with higher call rates and GenCall50 scores were observed ( $p$ $<0.001$ ) when filters F3 and F4 on flanking sequences were applied (Table 3). Furthermore, although comparison of SNP success rates across studies is not clear-cut due to the peculiarities of SNPs discovery and SNP reliability thresholds used, our overall SNP success rate averaged $87 \%$ if measured by the percentage of SNP with call rate $\geq 95 \%$, or $94 \%$ if measured by the proportion of SNPs with GeneTrain and GeneCall50 $\geq 0.4$ (Table 3). For the 288 SNPs selected with the most stringent filtering level F4, over $96 \%$ of them had GeneTrain and GeneCall50 $\geq 0.4$. These assay success rates are comparable to those obtained for the human [33] 
and barley [3] genomes. It is worth mentioning, however, that our considerably higher success rates when compared to other studies with highly heterozygous tree genomes, likely derives from the fact that the vast majority of the ESTs used were obtained from a relatively large sample with more than 21 unrelated diploid individuals (i.e. more than 42 sampled chromosomes) of E. grandis. More importantly, the pipeline filtered out SNPs that did not belong to the same exon by using the draft genome sequence for E. grandis, therefore avoiding failures due to SNP located in intron/exon junctions, a considerable drawback when developing SNPs from ESTs [51]. The impact of using a reference genome was likely responsible for the comparably high success rate $\geq$ $87 \%$ for the candidate genes SNPs for which no flanking sequence requirements could be applied. In summary, although we did not compare the reliability of SNPs designed without using a final selection step based on the reference genome, the simple comparison of our success rates with those obtained for comparably heterozygous tree species supports the value of having access to a reference genome sequence for successful large scale SNP development.

\section{SNP conversion rate was increased by selecting for conserved SNP flanking sequences}

An overall conversion rate of $66.1 \%$ was observed when genotype data for all 768 SNPs in a panel of 96 individuals of five species was considered. If only the 711 reliable SNPs are considered, the conversion rate increases to $71 \%$ which corresponds to the conversion rate of the top 288 SNPs developed after applying filter F4 on the SNP flanking sequences (Table 3 ). This conversion rate is equivalent to the one obtained for catfish SNPs developed from in silico ESTs after applying constraints on the number of ESTs and on the presence of minor allele sequences in the contig [51], and slightly higher than the conversion rates obtained for SNPs developed from in silico resources with no stringent filtering and assayed in analogous population samples of Pinus pinaster [10]. Interestingly, the proportion of polymorphic SNPs significantly increased $(\mathrm{p}=0.00221)$ when flanking sequence conservation of 60 bases was required. We hypothesize that the effect of flanking sequence conservation on polymorphism is not a direct one. It is partly a result of the higher SNP reliability but probably also due to an indirect effect of assaying a SNP surrounded by higher quality flanking sequences likely devoid of sequencing errors, and thus selected as more conserved. Such a SNP is therefore less likely to be a false SNP due to sequencing errors in one or more of the reads in the contig resulting in a better in silico assessment of polymorphism and consequently a more polymorphic one when assayed at the population level.

\section{Estimates of polymorphic SNPs within Eucalyptus species are conservative}

SNP polymorphism levels were also estimated for five species independently for which samples between 16 and 24 individuals (32 or 48 alleles) were genotyped (Table 4). The highest estimate was obtained for $E$. grandis (49.4\%) followed by E. camaldulensis (41.2\%) and E. urophylla (40.9\%). These estimates are relatively low when compared to other SNP development studies in forest trees especially bearing in mind the high nucleotide diversity in Eucalyptus. Estimates of MAF in SNP development studies are, however, strongly influenced by the sample size and by the genetic origin of the population [10]. For example, a sample size of 146 individuals (292 alleles) would be necessary to estimate an allele with frequency $0.05 \pm 0.025$ with $95 \%$ probability. The samples sizes used in our study were therefore not optimal to detect low frequency alleles at several SNPs that would otherwise be deemed polymorphic had we used a larger sample size. Furthermore, none of the individuals used to generate the ESTs were present in the genotyped panel. In fact several species were not even represented in the EST databases such as E. nitens and E. camaldulensis and even for E. globulus and E. urophylla the proportion of sequences used was very limited, less than $2 \%$ and $1 \%$ respectively. Therefore the estimates of the proportion of polymorphic SNPs in each species individually are conservative and should be taken as a lower bound estimate. Conversion rates will likely improve considerably by selecting SNPs from a sequence database built from a much wider representation of the diversity of each target species and validating in a larger panel of individuals.

As expected, the highest rate of polymorphic SNPs was observed for E. grandis, the predominant species in the EST database with over $96 \%$ of the sequences used for SNP discovery. Interestingly, however, E. camaldulensis showed the second highest conversion rate (41.2\%) despite the fact that not a single sequence was used for SNP discovery and that only 16 individuals, as compared to 24 in E. grandis, were genotyped. This result could be explained by a recent study that found E. camaldulensis with the highest nucleotide diversity among four Eucalyptus species, estimated at 1 SNP every 16 bp when amplicons in 23 genes were resequenced in 456 individuals from 93 populations [49]. In that same study several hundred individuals of E. globulus and E. nitens were also surveyed showing much lower nucleotide diversity, 31 and 33\% respectively, in an equivalently wide sample of individuals and populations. In our study these two species displayed the lowest proportion of polymorphic SNPs (22.2 and 25.5\%) (Table 4) and no statistically significant effect on the recovery of polymorphic SNPs was obtained by 
including at least one read from Maidenaria species $(E$. globulus or E. gunnii) in the contig, i.e. going from filter F1 to F2. Besides the ascertainment bias due to the very limited or nil representation of sequences in the EST databases, the lower proportion of polymorphic SNPs observed in these two species could be explained not only by their greater phylogenetic distance from $E$. grandis as compared to E. urophylla and E. camaldulensis but also by their intrinsically lower nucleotide diversity.

A substantial reduction in the proportion of simultaneously polymorphic SNP in two or more species was observed. The highest proportion of shared polymorphic SNPs was seen for the two and three-way combinations of E. grandis, E. urophylla and E. camaldulensis which agrees with their closest phylogenetic relationship. When Maidenaria species were included, however, the proportion of shared polymorphic SNPs dropped considerably to 14 to $18 \%$ and to $7.7 \%$ when all five species were contemplated together (Table 4 and Additional file 2). These results are consistent with in silico SNP sharing rates among four Eucalyptus species, estimated between 20 and $43 \%$ for 23 resequenced genes in much larger sample sizes [49]. In spite of the ascertainment bias that both in silico and assay-based estimates of shared polymorphic SNP suffer, these proportions suggest that a large number SNP pre-dating species separation will be available for assay development. From the practical standpoint this means that it is possible to develop a SNP array with informative SNPs across multiple Eucalyptus species. However the success of such an effort will strongly depend on their phylogenetic relationship and an extensive sampling of genome sequences of numerous individuals of each species. Furthermore all SNPs developed in our study were derived from expressed sequences, including 5 ' untranslated regions and exons. Kulheim et al. [49] showed a considerably higher SNP variability in introns when compared to exons in 23 genes in four Eucalyptus species. This result suggests that a higher SNP conversion rate could possibly be obtained in future SNP development efforts by screening SNPs derived from genomic sequences generated by massive NGS. On the other hand, however, the highest polymorphism of intronic and intergenic sequences should render more challenging the selection of SNPs with flanking sequences with no additional SNPs.

\section{SNP genotype calls match sequencing data and are correctly inherited in inter-specific crosses}

SNP validation was carried out two-ways: by parent-parent-offspring allele transmission test in two unrelated pedigrees and by shallow next-generation sequencing of a single individual of E. camaldulensis. The inheritance assessment showed an overall high rate of correct Mendelian transmission with almost $99 \%$ of correct genotype calls in the $E$. grandis $\times E$. urophylla pedigree and above $95 \%$ in the more diverse four-species pedigree. Reported genotyping inheritance miscall rates with the GGGT assay have been essentially zero in humans [33] and rarely reported for non-model plant genomes. Recently however, a global genotyping error rate of $0.54 \%$ in 188 SNPs was reported for Pinus pinaster [10] and between zero and $1 \%$ in polyploid wheat [52]. While the genotyping miscall rate of $1.2 \%$ in the E. grandis $\times E$. urophylla pedigree falls within expectations, the much higher $4.3 \%$ rate in the four-species family is probably a result of a reduced SNPs transferability to this more diverse genomic background. Alternatively these higher miscall rates could be due to paralogous genomic sequences being assayed, although generally this has not been a major problem with the GGGT even in complex plant genomes [27]. We decided to use inter-specific pedigrees for a rigorous SNP inheritance assessment considering that several envisaged application of SNP genotyping in Eucalyptus will contemplate progenies from wide interspecific crosses both for QTL mapping and the implementation of Genomic Selection. In both of these applications, however, a low proportion of genotype miscalls can be tolerated. This multi-species inheritance validation should be useful to reveal error-prone SNPs providing an additional selection criterion when developing a larger set of SNPs for genus wide genotyping in Eucalyptus.

Concordance between GGGT called genotypes and short-read sequencing was $86 \%$. This NGS-based SNP validation approach is practical, especially in highly heterozygous genomes where direct amplicon sequencing can be challenging, but evidently has some limitations as SNP sampling is strongly dependent on sequence coverage. Five divergent genotypes were called homozygous by the GGGT assay and inferred as heterozygous by sequencing. A manual curation of these discordant cases suggested that these could be due to paralogous genes being sequenced, although they could also be caused by sequencing errors. Two SNPs called as heterozygous by the GGGT but homozygous by sequencing correspond to false positives possibly due to the small number of reads available, only five for one SNP and six for the other (Additional file 3).

\section{SNP detection with MIRA could significantly enhance SNP conversion rates}

In an attempt to establish useful in silico predictors to guide future SNP development efforts, we further investigated the impact of the in silico variables used in the pipeline on SNP reliability measured by the GeneCall50 score and polymorphism by the MAF in E. grandis for 
which a large number of EST sequences was available. These variables were: 1 ) in silico estimated MAF; 2) the number of EST reads of a species at the SNP site; and 3) minimal distance to the next SNP site. GeneCall50 and MAF were modeled as binary response variables based on the established thresholds, i.e. SNPs were considered reliable if GeneCall50 $\geq 0.4$ and unreliable otherwise and polymorphic if MAF $\geq 0.05$ and monomorphic otherwise. Data were analyzed by means of a logistic regression [53] using each one of the explanatory variables above. The criterion for considering a SNP as polymorphic in silico was the presence of at least two reads with an alternative allele. The only significant explanatory variable for SNP reliability was the distance to the adjacent SNP site (Additional file 4), corroborating previous results. However the allele frequencies at SNP sites in the EST contigs (in silico MAF) and distance to the adjacent SNP site are not reliable predictors for SNP polymorphism. Interestingly, however, upon reviewing our SNP mining pipeline, we noticed that besides the final SNP calling by PolyBayes, an earlier SNP prediction is performed by MIRA, the EST clustering program. We therefore set to investigate the relative performance of both SNP calling approaches for the 696 genome-wide SNPs, reminding that all SNPs tested in the genotyping assay were predicted by PolyBayes with $\geq 99 \%$ probability. However, not all SNPs were tagged as such by MIRA. This inconsistency suggested the possible presence of SNP miscalls, which can be consequential to assay polymorphism. Out of the 696 SNPs considered, 632 were deemed reliable for $E$. grandis (GeneCall50 $\geq 0.4$ ) and were divided in two classes: in silico SNPs tagged (348) and not tagged (284) by MIRA. When SNPs in these two classes were classified for polymorphism, a clear trend emerged indicating that a significantly larger proportion of SNPs called in silico by both PolyBayes and MIRA were in fact polymorphic in the GGGT assay when compared by those called exclusively by PolyBayes $(p=0.00024)$. Considering SNPs predicted only with PolyBayes the conversion rate was $43.4 \%$. For SNPs called by both PolyBayes and MIRA the conversion rate was $58.1 \%$. This result could be explained by the way that the two SNP calling algorithms operate. Polybayes likely suffered from the relatively large number of ESTs obtained in GeneBank for which no base quality values were available. In these cases an arbitrary $Q$ value of 15 was assigned, a procedure that later impacted the estimate of SNP probability. MIRA, on the other hand, uses a sliding window of sequence quality instead of a single column, a strategy that favors the estimation of base quality and consequently an enhanced accuracy in SNP detection. This unexpected result suggests that in future SNP development efforts SNP tagging by MIRA could lead to higher SNP conversion rates.

SNPs are reliable across Eucalyptus species and subgenera Very few studies to date assessed the transferability of the same SNP genotyping array across a wide range of species within the same genus. In grape, transferability of SNPs assayed by the SNPlex ${ }^{\mathrm{TM}}$ Genotyping System (Applied Biosystems Inc.) averaged 18.8\% across V. vinifera wild forms and only $2.3 \%$ when genotyping nonvinifera Vitis species. Only 4 SNPs out of 137 were polymorphic (MAF values $\geq 0.30$ ), in non-vinifera Vitis species [35]. In the genus Picea in 279 resequenced genes that had at least one SNP in each of white spruce $(P$. glauca) and black spruce (P. mariana), only $4.7 \%$ of the observed SNPs were shared between the two species, requiring the development of separate 768 SNP arrays for each species. Recently, NGS of reduced representation libraries from 10 cultivated $V$. vinifera varieties and 6 wild Vitis species was used to develop a selected set of 8,898 SNPs and $24.3 \%$ of the were shared between $V$. vinifera and wild Vitis species [13]. In bovines when the 50K BovineSNP50 assay was applied to a set of DNA samples from six other species within Bovinae, including two from different genera, over $96 \%$ of the SNP produced genotype calls for at least five of the species including the four species within the genus Bos but only between 1 and $5 \%$ of the SNP that produced genotype calls were polymorphic despite the relatively recent divergence (1-5 Mya) between these species and Bos taurus [50]. In our study SNP genotyping reliability rates were high across nine species belonging to three sections within subgenus Symphomyrtus with more that $83 \%$ of the SNPs with call rates $\geq 95 \%$ and GeneCall 50 $\geq 0.4$ between $92 \%$ and $95 \%$. Transferability rates were still satisfactory when going across subgenera with over $50 \%$ of the SNPs showing call rates $\geq 95 \%$ in two more distant species (Table 5). Estimates of divergence times among eucalypt lineages are still controversial. Those based on climatic and tectonic events suggest that the radiation of species within sections ( $<2$ Mya) and sections within subgenus Symphyomyrtus (5-10 Mya) [54] are much more recent than estimates from molecular dating (5-10 Mya) and (13-36 Mya) respectively [55]. The relatively high transferability rates of our SNP panel across sections of Symphyomyrtus and even across distinct subgenera most likely agrees with a more recent species divergence and/or the maintenance of large population sizes over time and suggests that a reasonable proportion of SNPs developed for species of Symphyomyrtus pre-date the divergence from other subgenera and could be useful for genetic analysis in more distant species of Eucalyptus. 


\section{Conclusion and perspectives}

We have shown that a large number of SNPs assayed by the GGGT can be successfully developed from in silico sequence resources for a complex genome with very high nucleotide diversity. Using a systematic approach we have also shown that the application of stringent criteria on SNP flanking sequences in silico provides enhanced SNP reliability and good conversion rates across multiple species despite the absence of several of them in the EST collection used for SNP discovery. Although this might be a distinctiveness of Eucalyptus, where species radiation is probably recent, this study suggests that the Golden Gate assay could be a practical SNP genotyping method to carry out SNP-based population genomics studies in other outbreeding tropical tree genera. Nevertheless, SNP conversion success will be strongly dependent on having a representative and deep collection of sequences of the target species and a robustly selective SNP discovery pipeline including a moderate quality draft genome sequence now within reach for many species using third-generation singlemolecule real-time DNA sequencing [56].

This study has provided the groundwork for a larger scale effort to develop a significantly larger SNP array for Eucalyptus. Following the successful reports published for a number of species to date $[2,13,50]$, we are now using deep sequencing of reduced representation libraries (RRLs) to develop a large numbers of informative SNPs across the main species of Eucalyptus planted worldwide. SNPs discovered in diverse RRLs will provide exceptional opportunities to discover ancestry informative SNPs for genome-wide phylogenetic reconstructions and dating at different levels in Eucalyptus and related genera. Finally, a higher density SNP platform could be instrumental to implement Genomic Selection in Eucalyptus [57] although datapoint costs have to drop by an order of magnitude to become economically viable in tree breeding programs that encompass tens of thousands of samples. The current DArT marker array provides adequate density and genome-coverage at unbeatable costs [46]. However co-dominant SNPs would provide an added advantage to estimate haplotypes and allow the inclusion of non-additive effects in the predictive models, thereby increasing the expected accuracy of genomic predicted breeding values.

\section{Methods}

\section{EST resources}

Three EST datasets were used in this work. The first one is a Sanger 5' sequenced set of 101,240 ESTs from E. grandis, E. globulus, E. pellita and E. urophylla generated in the Genolyptus project [58]. The second one is a Sanger set of 34,801 sequences downloaded from dbEST that included 1,990 sequences from E. grandis, 19,860 from E. gunnii, 13,863 from E. globulus and the remaining from other species. The third was a dataset of $1,028,654$ ESTs from NCBI SRA [accession SRX000427] generated from 21 individual trees of $E$. grandis using the 454 pyrosequencing technology [18]. These are the final numbers of sequences following removal of possible contaminations with vector, linker and adaptors using SeqClean and Crossmatch [59]. Poly (A/T) tails were trimmed, retaining a short $6-10 \mathrm{bp}$ sequence to get quality ESTs for subsequent clustering, alignment and assembly. To assist in avoiding over-trimming of the hypothesized polyA/T site, an iterative scan for such homo-oligomers was implemented with est2assembly [60]. This routine also contributes to minimize errors produced by the 454 pyrosequencing methodology regarding detection of homopolymer sequences. Usersupplied files with Phred scores [61] when available, were used as a measure of sequence quality in the pipeline. EST datasets downloaded from dbEST without associated quality information had their scores internally computed during the alignment process using Phrap [62].

\section{EST clustering and alignment}

ESTs derived from each Eucalyptus species and with each technology (Sanger or 454) were grouped separately into clusters, each one expected to correspond to a single DNA segment and then aligned with the help of the user-supplied and internally computed base quality information using Phrap for Sanger reads or Newbler for 454 reads. Clustering was carried out using an algorithm based on the dissimilarity measures criteria with subword comparisons. Evaluation of the suitability of string dissimilarity measures for EST clustering was reported earlier [63]. The wcd EST clustering system [64] used in this study is an open source clustering system that provides efficient implementation of different dissimilarity measures, heuristics for speeding up clustering, a pre-clustering booster based on suffix arrays, as well as parallelized implementations based on MPI and Pthreads. The wcd EST clustering can be used to cluster large sets of mixed EST and RNA sequences, and is adaptable to shorter length error-prone sequencing technologies. For alignment, conservative Phrap parameters were applied to prevent misalignments in the resulting clusters and data quality information was used to ensure that maximum numbers of individual sequences were retained. A similar Phrap alignment investigation approach was used when processing sequences for loblolly pine SNP identification [65]. Clustering of the 454 sequences was carried out using Newbler 2.3 (Roche-applied.science.com). Aligned sequences in clusters comprised two or more sequences and could be a combination of one or more contigs. Sequences not aligned within a cluster corresponded to ESTs that were considered as unique within each dataset, 
i.e. singlets. Contigs and singlets were joined into one single FASTA file and another single file with quality data information was obtained. Each sequence in the FASTA file was identified in a third accompanying file which contained information about the Eucalyptus species of origin.

\section{EST assembly and identification of candidate SNPs}

A general pipeline was put in place based on the software MIRA 2 - Whole Genome Shotgun and EST Sequence Assembler V2.9.25 with the enhanced 454 and Illumina/Solexa support. MIRA is an EST sequence assembler that specializes in reconstruction of pristine mRNA transcripts, while at the same time detecting and classifying single nucleotide polymorphisms (SNPs) occurring in different variations thereof [66]. MIRA combines a redundancy based approach with a symbolic pattern analyzer developed for recognition of column discrepancies in sequence alignments to allow detection of SNPs. In the algorithm implemented by MIRA the decision on whether discrepancies between similar EST sequences are significant or not relies more on the underlying quality information data. Under those circumstances, even a discrepancy caused by a single base in a single column of an alignment can be seen as a hint for a SNP site, i.e., if the base probability values of the bases in the immediate area are high and do not allow an alternative sequencing error hypothesis. The most important criterion adjudicating a SNP was therefore the group qualities of the bases in the immediate area of the SNP site - a SNP block - that can be calculated for different bases in a column. To investigate if group qualities of the bases in the vicinity of the SNP site could serve as a good predictor of true SNPs, SNPs from each one of those tags together with other nontagged sites were selected from the list generated by MIRA. The selected predictors representing this classifier are a combination of two measures provided by the MIRA Assembler at the SNP site: the major allele score and the minor allele score. The minor allele is the allele occurring in the minority of the sequences at the SNP site, while the other is called the major allele. All the tagged or non-tagged SNP sites from the previous assembly step were submitted to an in silico evaluation for assessing the probability that a given site was polymorphic. SNP prediction was carried out using PolyBayes version 3.0 [67] with a p prior of 0.01 corresponding to an expected mean frequency of one SNP every 100 nucleotides and putative SNPs called with a stringent cutoff probability of $\mathrm{P}_{\mathrm{SNP}} \geq 0.99$.

\section{SNP selection for GGGT assay design}

Following the Polybayes probability and SNP vicinity scores provided by the alignment, a set of parameters were defined to construct filters to select SNPs for the GGGT assay design. Initially all SNPs had to have at least 60 bases available on each SNP side to allow designing allele and locus specific oligos for the GGGT assay. Additionally the following parameters were used: (1) the in silico estimated minor allele frequency (MAF) at the SNP site; (2) the number of EST reads by target species aligned at each SNP site; and (3) the presence of additional SNPs along the SNP flanking sequences. The impact of these parameters on SNP reliability and polymorphism validated by the GGGT assay was evaluated by sequentially implementing increasingly stringent in silico SNP filtering levels as follows:

\section{Filter FO}

bi-allelic SNPs with $\geq 5$ reads on SNP position, and a minimum of one read with the alternative base;

Filter F1

bi-allelic SNPs with $\geq 5$ reads on SNP position and a Minor Allele Frequency (MAF) $\geq 0.2$;

\section{Filter F2}

same as $\mathrm{F} 1$ plus a minimum of one read derived from E. globulus or E. gunnii; these species belong to the section Maidenaria, phylogenetically separate from E. grandis, E. urophylla and E. pellita that belong to section Latoangulatae;

\section{Filter F3}

same as F2 plus a minimum of 100 bases on each SNP side without repetitive elements and a minimum of 20 bases flanking the SNP on each side without any additional SNPs in the contig;

\section{Filter F4}

same as F3 but increasing to a minimum of 60 bases flanking the SNP on each side without any additional SNPs in the contig.

As the SNPs were derived from cDNA sequences, the occurrence of intron/exon border in the flanking sequences upon which the oligos are later designed, may cause significant genotyping failure [51]. To mitigate this problem an additional analysis based on the Exonerate program [68] using the est2genome model, was implemented on all SNPs that passed one or more of the five filters. Only SNPs that had $30 \mathrm{bp}$ on each side belonging to the same exon were selected. The $4.5 \mathrm{X}$ draft genomic assembly of Eucalyptus grandis used as reference was downloaded from the EUCAGEN (Eucalyptus Genome Network) site at http://Eucalyptusdb.bi.up.ac.za.

From each one of the five filtered subsets of SNPs a random sample between $\sim 300$ and 600 SNPs was derived to be subsequently submitted to the Assay Design Tool (ADT) made available by Illumina with a target of having between 96 and 288 SNPs to be effectively designed and validated. Each SNP receives a SNP score that is a predictor of the likelihood of genotyping success using the GGGT assay. ADT generates scores 
for each SNP that could vary from 0 to 1 . According to the Illumina standard recommendations, a SNP with score $\geq 0.6$ has a high probability to be converted into a successful genotyping assay. Based on the ADT scores returned by Illumina, a subset of SNPs to be included in the SNP bead array was randomly selected from the final set of SNPs obtained with a more stringent ADT $\geq$ 0.8 for each one of the five filter treatments.

\section{SNPs selected in candidate genes}

A list of 42 genes described in the literature as being putatively associated with relevant wood phenotypes in Eucalyptus was compiled. These were genes involved in lignin biosynthesis $[69,70]$, genes derived from expression studies of wood formation using microarrays $[71,72]$, expression-QTL mapping $[73,74]$ and association studies [48]. GenBank accession numbers for these genes or their names as described in the respective papers were used to select and assemble ESTs from the databases for SNPs discovery. All SNPs that satisfied the standard requirement of 60 bases of available sequence on each SNP side and at least two reads with alternative bases at the SNP position (i.e a more relaxed selection than Filter F0 which required $\geq 5$ reads at the SNP position) were submitted to the Illumina assay design tool and those with ADT score $\geq 0.6$ were subsequently selected to populate the bead pool array.

\section{Plant material and DNA extraction}

Population samples of unrelated trees of each one of the five most widely planted and bred species worldwide were used for SNP reliability, inter-specific transferability and polymorphism assessment. All these five species belong to the same subgenus Symphomyrtus but to different sections. Sample sizes were N $=24$ for E. grandis (section Latoangulatae), $\mathrm{N}=24$ for E. globulus (section Maidenaria), $\mathrm{N}=16$ for E. urophylla (section Latoangulatae), $\mathrm{N}=16$ for E. camaldulensis (section Exsertaria) and $\mathrm{N}=16$ for E. nitens (section Maidenaria), totaling 96 individuals. For E. grandis, twelve trees from each of two different provenances were sampled, Atherton $\left(17^{\circ}\right.$ $\left.15^{\prime} \mathrm{S} 145^{\circ} 28^{\prime} \mathrm{E}\right)$ and Coffs Harbor $\left(30^{\circ} 18^{\prime} \mathrm{S} 153^{\circ} 07^{\prime} \mathrm{E}\right)$; for E. globulus twelve trees were from Jeeralang $\left(38^{\circ} 24^{\prime} \mathrm{S}\right.$ $\left.146^{\circ} 28^{\prime} \mathrm{E}\right)$ and twelve from Flinders Island $\left(40^{\circ} 00^{\prime} \mathrm{S} 148^{\circ}\right.$ $\left.07^{\prime} \mathrm{E}\right)$; for E. urophylla all trees were from Flores Island $\left(8^{\circ} 39^{\prime} \mathrm{S} 122^{\circ} 15^{\prime} \mathrm{E}\right)$, for E. camaldulensis all trees were from Walsh River $\left(17^{\circ} 17^{\prime} \mathrm{S} 144^{\circ} 88^{\prime} \mathrm{E}\right)$ and for E. nitens trees were from Eastern Ebor $\left(30^{\circ} 24^{\prime} \mathrm{S} 152^{\circ} 29^{\prime} \mathrm{E}\right)$. Small samples of $\mathrm{N}=8$ individuals were also genotyped for other six species of more limited world relevance to provide a preliminary assessment of SNP transferability across a wider inter-specific range and across two additional subgenera. These were: E. dunnii, E. saligna, E. tereticornis, these four also belonging to the subgenus
Symphomyrtus, and E. cloeziana and E. pilularis belonging to two different subgenera, Idiogenes and Eucalyptus, respectively. DNA extractions from fresh expanded leaves were carried out as described earlier using a modified $C T A B$ procedure [75] and quantified using the PicoGreen assay (Invitrogen, Carlsbad, CA, USA) in a Nanodrop 3300 micro-volume fluorospectrometer and standardized to Illumina-specified concentrations for SNP genotyping (50-100 ng/ $\mu \mathrm{L})$.

\section{SNP genotyping}

Selected SNPs were used to construct an Illumina bead array of 768 SNPs based on the GoldenGate assay (Illumina Inc., San Diego, California). Out of these 768 SNPs, 696 were distributed across the five SNP selection filters while 72 SNPs were derived from specific candidate genes. Genotyping was performed using an Illumina BeadStation 500 GX (Illumina, San Diego, CA) at the Genome facility of the Interdisciplinary Center for Biotechnology Research (ICBR) of the University of Florida using the protocol described earlier [76]. SNP data were analyzed using GenomeStudio V2009.2 Genotyping module 1.5.16 (Illumina, San Diego, CA) that clusters and calls the data automatically, allowing visualization of the data directly for downstream analysis. Call rates, GeneTrain and GenCall scores were exported using the GenomeStudio internal Locus Summary Report Tool for the subsequent analysis of SNP reliability and polymorphism.

\section{SNP genotyping reliability}

SNP reliability was evaluated by the GeneTrain and the GeneCall scores. The GenTrain score, a statistics with a value between 0 and 1 , was estimated for each SNP to assess the quality of the shapes of the genotype clusters (homozygous and heterozygous) and their relative distance to each other. A GenCall score, estimated for each datapoint (SNP $\times$ individual sample), is designed to rank particular DNA samples or SNP loci and is obtained by the product of the GenTrain Score and a data-to-Bayesian-model fit score as implemented by the Genome Studio software. Genotypes with lower GenCall scores are located further from the center of the genotype cluster and have a lower reliability. To assess the reliability of each individual SNP the GenCall scores for all typed samples for each SNP were used to estimate a GeneCall50 (GC50) score that corresponds to the 50th percentile (median) of the distribution of the GenCall scores for that SNP. A GeneCall50 score threshold $\geq$ 0.40 was used to declare a SNP reliable. SNPs genotyping performance was additionally assessed by the call rate for each SNP using a GeneCall score cutoff $\geq 0.25$ for each datapoint $(\mathrm{SNP} \times$ Individual sample) following the Illumina recommended threshold for GGGT [76]. 
Reliability parameters were evaluated consolidating genotyping data for all species that had 16 or more individuals genotyped and for each species separately.

\section{SNP polymorphism information content}

Polymorphism was evaluated by the conventional MAF (Minimum Allele Frequency) parameter. A SNP was considered polymorphic if MAF $\geq 0.05$. Polymorphism was evaluated consolidating data for all samples together, irrespective of species, and also for each species separately but only for those species that had at least 16 samples (32 chromosomes) analyzed. Species for which only eight individuals were analyzed could not provide acceptable estimates of polymorphism level.

\section{Assessment of the in silico variables on SNP reliability and polymorphism}

Goodness-of-fit for normality tests were carried out on GeneTrain and GeneCall50 scores estimated for each SNP. Mann-Whitney non-parametric tests were carried out to assess the impact of the SNP filtering levels (F0 through F4) on GeneTrain and GeneCall50 scores. Furthermore, using a GeneTrain score $\geq 0.4$, a GC50 score $\geq 0.4$, a call rate $\geq 95 \%$ as thresholds for declaring a reliable SNP and a MAF $\geq 0.05$ as a threshold to declare a polymorphic SNP, pair-wise Chi-square Pearson contingency tests were used to assess the impact of the five filtering levels on the final proportions of SNPs declared as being reliable and polymorphic. The final SNP conversion rate estimated by the proportion of polymorphic SNPs (MAF $\geq 0.05$ ) within the ones deemed reliable (GeneTrain score $\geq 0.4$ and GeneCall50 score $\geq 0.4$ ) was estimated for the whole data set and for each one of the main five species separately.

\section{Inheritance-based SNP validation}

Heritability of SNP allelic transmission was calculated for each SNP using data from a total of 48 parent-parent-offspring trios involving different Eucalyptus species as parents. Both parents and 24 offspring individuals were randomly selected from each one of two segregating populations, one derived from a E. grandis $\times E$. urophylla interspecific cross and the second one from a four-species cross involving a $E$. dunni $\times E$. grandis male parent and a E. urophylla $\times$ E. globulus female parent. Heritability was estimated using Genome Studio internal heritability report tool by counting the proportion of correct allelic transmissions for each set of 24 trios.

\section{Sequence-based validation of SNP genotypes}

Genotypes for queried SNPs were validated for a sample of $E$. camaldulensis by comparing the GoldenGate called genotypes with NGS based genotypes derived from shotgun genomic reads (Illumina $2 \times 76$ bases pairedend reads) providing an estimated $2 \mathrm{X}$ coverage of the 630 Mbp Eucalyptus genome. Sequence based genotypes were called only for SNP positions that had at least five clustered reads on the reference genome and sequence quality $Q \geq 20$. A sample size of 5 reads provides an expected probability of 0.9375 of detecting at least one read with the alternative allele if the genotype is heterozygous. Sequence based genotypes were declared based on a simple binomial test where a null hypothesis of an expected 1:1 ratio of the read counts was set. When the null hypothesis was not rejected a heterozygous genotype was declared. Rejection of the null hypothesis, on the other hand, led to the inference of a homozygous genotype at the SNP.

\section{Additional material}

Additional file 1: Information on the 768 Eucalyptus SNPs developed. SNPS are organized in two separate lists, one with the 696 genome-wide SNPS selected with the five in silico sequential filtering levels (locus names start with F0 through F4 - see methods) and a second one with the 72 candidate gene SNPs. Information on the SNP type, SNP flanking sequences used for GGGT assay design, ADT score, Call rate, Minor allele frequency, GeneTrain score and GeneCall50 score is provided, estimated from the 96 DNA samples typed of the five main Eucalyptus species (E. grandis, E. urophylla, E. globulus, E. nitens and E. camaldulensis). Contig sequence used for SNP discovery and BLAST annotation are also provided.

Additional file 2: Supplementary material S2. Counts and percentages of polymorphic SNPS (MAF $\geq 0.05$ ) from a total of 711 reliable SNPs in all 26 combinations of the five main Eucalyptus species surveyed.

Additional file 3: Supplementary material S3. Results of the NGSbased validation of SNP genotypes. Golden Gate Genotype calls (GGGT genotype) for a Eucalyptus camaldulensis individual were compared to sequence-based genotypes inferred from Illumina short read sequencing based on a binomial test where a null hypothesis of an expected 1:1 ratio of the read counts was set. When the null hypothesis was not rejected a heterozygous genotype was declared. Rejection of the null hypothesis, on the other hand, led to the inference of a homozygous genotype at the SNP. Shaded in grey are the seven SNPs genotypes that showed divergent results between GGGT and NGS genotype calls.

Additional file 4: Supplementary material S4. Results of logistic regression of the in silico variables used in the SNP discovery and filtering pipeline in E. grandis on SNP Reliability and SNP Polymorphism treated as binary characters (reliability defined by GeneCall50 $\geq 0.4$ and polymorphism by MAF $\geq 0.05$ ).

\section{Acknowledgements}

This work was mainly supported by the Brazilian Ministry of Science and Technology through CNPq grant 577047/2008-6 and by additional funds from EMBRAPA Macroprogram 2 project grant 02.07.01.004 and BiotecSur UE 127118 grant; BML and DAF were sponsored by a doctoral and postdoctoral fellowships from FAPESP and CNPq respectively; GJPJr and DG have been awarded research fellowships from CNPq. We acknowledge the earlier contributions of Evandro Novaes and Roberto Togawa with SNP data mining using approaches that were not followed up in this work. We also acknowledge the excellent SNP genotyping service carried out by Ginger Clark, Scientific Research Manager of the Genetic Analysis Lab at the ICBR (Interdisciplinary Center for Biotechnology Research) of the University of Florida. 


\section{Author details}

'EMBRAPA Genetic Resources and Biotechnology - Estação Parque Biológico, final W5 norte, Brasilia, Brazil. ${ }^{2}$ Genomic Sciences Program - Universidade Catolica de Brasília- SGAN, 916 modulo B, 70790-160 Brasília - DF, Brazil. ${ }^{3}$ School of Forest Resources and Conservation, Genetics Institute, University of Florida, PO Box 110410, Gainesville, USA. ${ }^{4}$ Department of Genetics Universidade de São Paulo - ESALQ/USP - Av. Pádua Dias, 11 - Caixa Postal 9 13418-900 Piracicaba, SP, Brazil.

\section{Authors' contributions}

DG was responsible for project conception, statistical analyses, manuscript writing, overall supervision of project execution and funding; OSJr was involved in the conception and development of the SNP discovery and design pipeline, and contributed to statistical analyses and manuscript preparation; MK contributed to SNP genotyping; BML contributed to DNA preparation; DAF was responsible for candidate gene selection and helped with data analysis; GJPJr conceived the bioinformatics pipeline development and was responsible for overall bioinformatics supervision. All authors have read and approved the final manuscript.

Received: 21 December 2010 Accepted: 14 April 2011

Published: 14 April 2011

\section{References}

1. Atwell S, Huang YS, Vilhjalmsson BJ, Willems G, Horton M, Li Y, Meng D, Platt A, Tarone AM, Hu TT, et al: Genome-wide association study of 107 phenotypes in Arabidopsis thaliana inbred lines. Nature 2010, 465(7298):627-631

2. Hyten DL, Cannon SB, Song QJ, Weeks N, Fickus EW, Shoemaker RC, Specht JE, Farmer AD, May GD, Cregan PB: High-throughput SNP discovery through deep resequencing of a reduced representation library to anchor and orient scaffolds in the soybean whole genome sequence. Bmc Genomics 2010, 11:38.

3. Close T, Bhat P, Lonardi S, Wu Y, Rostoks N, Ramsay L, Druka A, Stein N, Svensson J, Wanamaker $S$, et al: Development and implementation of high-throughput SNP genotyping in barley. BMC Genomics 2009, 10(1):582.

4. Buckler ES, Holland JB, Bradbury PJ, Acharya CB, Brown PJ, Browne C, Ersoz E, Flint-Garcia S, Garcia A, Glaubitz JC, et al: The Genetic Architecture of Maize Flowering Time. Science 2009, 325(5941):714-718.

5. Zhu C, Gore M, Buckler ES, Yu J: Status and Prospects of Association Mapping in Plants. The Plant Genome 2008, 1(1):5-20.

6. Rafalski A: Applications of single nucleotide polymorphisms in crop genetics. Current Opinion in Plant Biology 2002, 5(2):94-100.

7. Luikart G, England PR, Tallmon D, Jordan S, Taberlet P: The power and promise of population genomics: From genotyping to genome typing. Nature Reviews Genetics 2003, 4(12):981-994.

8. Muchero W, Diop N, Bhat P, Fenton R, Wanamaker S, Pottorff M, Hearne S, Cisse N, Fatokun C, Ehlers J, et al: A consensus genetic map of cowpea [Vigna unguiculata (L) Walp.] and synteny based on EST-derived SNPs. Proceedings of the National Academy of Sciences of the United States of America 2009, 106(43):18159-18164.

9. Eckert A, Pande B, Ersoz E, Wright M, Rashbrook V, Nicolet C, Neale D: Highthroughput genotyping and mapping of single nucleotide polymorphisms in loblolly pine (Pinus taeda L.). Tree Genetics \& Genomes 2009, 5(1):225-234

10. Lepoittevin C, Frigerio JM, Garnier-Gere P, Salin F, Cervera MT, Vornam B, Harvengt L, Plomion C: In vitro vs in silico detected SNPs for the development of a genotyping array: what can we learn from a nonmodel species? PLoS One 2010, 5(6): 11034.

11. Pavy N, Pelgas B, Beauseigle Sp, Blais S, Gagnon F, Gosselin I, Lamothe M, Isabel N, Bousquet J: Enhancing genetic mapping of complex genomes through the design of highly-multiplexed SNP arrays: application to the large and unsequenced genomes of white spruce and black spruce. BMC genomics 2008, 9(1):21.

12. Wegrzyn JL, Eckert AJ, Choi M, Lee JM, Stanton BJ, Sykes R, Davis MF, Tsai CJ, Neale DB: Association genetics of traits controlling lignin and cellulose biosynthesis in black cottonwood (Populus trichocarpa, Salicaceae) secondary xylem. New Phytologist 2010, 188(2):515-532

13. Myles S, Chia JM, Hurwitz B, Simon C, Zhong GY, Buckler E, Ware D: Rapid Genomic Characterization of the Genus Vitis. Plos One 2010, 5(1):e8219.
14. Vezzulli S, Troggio M, Coppola G, Jermakow A, Cartwright D, Zharkikh A, Stefanini M, Grando MS, Viola R, Adam-Blondon AF, et al: A reference integrated map for cultivated grapevine (Vitis vinifera L.) from three crosses, based on 283 SSR and 501 SNP-based markers. Theoretical and Applied Genetics 2008, 117(4):499-511.

15. Chagne D, Gasic K, Crowhurst RN, Han Y, Bassett HC, Bowatte DR, Lawrence TJ, Rikkerink EHA, Gardiner SE, Korban SS: Development of a set of SNP markers present in expressed genes of the apple. Genomics 2008, 92(5):353-358.

16. Ganal MW, Altmann T, Roder MS: SNP identification in crop plants. Current Opinion in Plant Biology 2009, 12(2):211-217.

17. Nordborg M, Weigel D: Next-generation genetics in plants. Nature 2008, 456(7223):720-723

18. Novaes E, Drost DR, Farmerie WG, Pappas GJ Jr, Grattapaglia D, Sederoff RR, Kirst M: High-throughput gene and SNP discovery in Eucalyptus grandis, an uncharacterized genome. BMC Genomics 2008, 9:312.

19. Barbazuk WB, Emrich SJ, Chen HD, Li L, Schnable PS: SNP discovery via 454 transcriptome sequencing. Plant Journal 2007, 51(5):910-918.

20. van Orsouw NJ, Hogers RCJ, Janssen A, Yalcin F, Snoeijers S, Verstege E, Schneiders $H$, van der Poel $H$, van Oeveren J, Verstegen $H$, et al: Complexity Reduction of Polymorphic Sequences (CRoPS (TM)): A Novel Approach for Large-Scale Polymorphism Discovery in Complex Genomes. Plos One 2007, 2(11).

21. Van Tassell CP, Smith TPL, Matukumalli LK, Taylor JF, Schnabel RD, Lawley CT, Haudenschild CD, Moore SS, Warren WC, Sonstegard TS: SNP discovery and allele frequency estimation by deep sequencing of reduced representation libraries. Nature Methods 2008, 5(3):247-252.

22. Okou DT, Steinberg KM, Middle C, Cutler DJ, Albert TJ, Zwick ME: Microarray-based genomic selection for high-throughput resequencing. Nature Methods 2007, 4(11):907-909.

23. Gnirke A, Melnikov A, Maguire J, Rogov P, LeProust EM, Brockman W, Fennell T, Giannoukos G, Fisher S, Russ C, et al: Solution hybrid selection with ultra-long oligonucleotides for massively parallel targeted sequencing. Nature Biotechnology 2009, 27(2):182-189.

24. Mamanova L, Coffey AJ, Scott CE, Kozarewa I, Turner EH, Kumar A Howard E, Shendure J, Turner DJ: Target-enrichment strategies for nextgeneration sequencing. Nature Methods 2010, 7(2):111-118.

25. Parameswaran P, Jalili R, Tao L, Shokralla S, Gharizadeh B, Ronaghi M, Fire AZ: A pyrosequencing-tailored nucleotide barcode design unveils opportunities for large-scale sample multiplexing. Nucleic Acids Research 2007, 35(19).

26. Baird NA, Etter PD, Atwood TS, Currey MC, Shiver AL, Lewis ZA, Selker EU, Cresko WA, Johnson EA: Rapid SNP Discovery and Genetic Mapping Using Sequenced RAD Markers. Plos One 2008, 3(10).

27. Hyten DL, Song Q, Choi IY, Yoon MS, Specht JE, Matukumalli LK, Nelson RL, Shoemaker RC, Young ND, Cregan PB: High-throughput genotyping with the GoldenGate assay in the complex genome of soybean. Theoretical and Applied Genetics 2008, 116(7):945-952.

28. Yan JB, Yang XH, Shah T, Sanchez-Villeda H, Li JS, Warburton M, Zhou Y, Crouch JH, Xu YB: High-throughput SNP genotyping with the GoldenGate assay in maize. Molecular Breeding 2010, 25(3):441-451

29. Muchero W, Diop NN, Bhat PR, Fenton RD, Wanamaker S, Pottorff M, Hearne S, Cisse N, Fatokun C, Ehlers JD, et al: A consensus genetic map of cowpea [Vigna unguiculata (L) Walp.] and synteny based on EST-derived SNPs. Proceedings of the National Academy of Sciences of the United States of America 2009, 106(43):18159-18164.

30. Pavy N, Pelgas B, Beauseigle S, Blais S, Gagnon F, Gosselin I, Lamothe M, Isabel N, Bousquet J: Enhancing genetic mapping of complex genomes through the design of highly-multiplexed SNP arrays: application to the large and unsequenced genomes of white spruce and black spruce. BmC Genomics 2008, 9.

31. Eckert AJ, Pande B, Ersoz ES, Wright MH, Rashbrook VK, Nicolet CM, Neale DB: High-throughput genotyping and mapping of single nucleotide polymorphisms in loblolly pine (Pinus taeda L.). Tree Genetics \& Genomes 2009, 5(1):225-234.

32. Lijavetzky D, Cabezas JA, Ibanez A, Rodriguez V, Martinez-Zapater JM: High throughput SNP discovery and genotyping in grapevine (Vitis vinifera L.) by combining a re-sequencing approach and SNPlex technology. BMC Genomics 2007, 8:424.

33. Shen R, Fan J, Campbell D, Chang W, Chen J, Doucet D, Yeakley J, Bibikova M, Wickhamgarcia E, McBride C: High-throughput SNP genotyping on universal bead arrays. Mutation Research/Fundamental and Molecular Mechanisms of Mutagenesis 2005, 573(1-2):70-82. 
34. Brockman W, Alvarez P, Young S, Garber M, Giannoukos G, Lee WL, Russ C, Lander ES, Nusbaum C, Jaffe DB: Quality scores and SNP detection in sequencing-by-synthesis systems. Genome Research 2008, 18(5):763-770.

35. Vezzulli S, Micheletti D, Riaz S, Pindo M, Viola R, This P, Walker MA, Troggio M, Velasco R: A SNP transferability survey within the genus Vitis. Bmc Plant Biology 2008, 16(8):128.

36. Grattapaglia D, Kirst M: Eucalyptus applied genomics: from gene sequences to breeding tools. New Phytologist 2008, 179(4):911-929.

37. Steane DA, Nicolle D, Vaillancourt RE, Potts BM: Higher-level relationships among the eucalypts are resolved by ITS-sequence data. Australian Systematic Botany 2002, 15:49-62.

38. Grattapaglia D, Ribeiro VJ, Rezende GD: Retrospective selection of elite parent trees using paternity testing with microsatellite markers: an alternative short term breeding tactic for Eucalyptus. Theor Appl Genet 2004, 109(1):192-199

39. Byrne M, Murrell JC, Allen B, Moran GF: An integrated genetic linkage map for eucalypts using RFLP, RAPD and isozyme markers. Theoretical and Applied Genetics 1995, 91:869-875.

40. Brondani R, Williams E, Brondani C, Grattapaglia D: A microsatellite-based consensus linkage map for species of Eucalyptus and a novel set of 230 microsatellite markers for the genus. BMC Plant Biology 2006, 6(1):20.

41. Thamarus K, Groom K, Murrell J, Byrne M, Moran G: A genetic linkage map for Eucalyptus globulus with candidate loci for wood, fibre and floral traits. Theor Appl Genet 2002, 104:379-387.

42. Grattapaglia D, Bertolucci FL, Penchel R, Sederoff RR: Genetic mapping of quantitative trait loci controlling growth and wood quality traits in Eucalyptus grandis using a maternal half-sib family and RAPD markers. Genetics 1996, 144(3):1205-1214.

43. Freeman JS, Whittock SP, Potts BM, Vaillancourt RE: QTL influencing growth and wood properties in Eucalyptus globulus. Tree Genetics \& Genomes 2009, 5(4):713-722.

44. Junghans DT, Alfenas AC, Brommonschenkel SH, Oda S, Mello EJ, Grattapaglia D: Resistance to rust ( Puccinia psidii Winter) in eucalyptus: mode of inheritance and mapping of a major gene with RAPD markers. Theor Appl Genet 2003, 108(1):175-180.

45. Thamarus K, Groom K, Bradley A, Raymond CA, Schimleck LR, Williams ER, Moran GF: Identification of quantitative trait loci for wood and fibre properties in two full-sib properties of Eucalyptus globulus. Theor App/ Genet 2004, 109(4):856-864.

46. Sansaloni CP, Petroli CD, Carling J, Hudson CJ, Steane DA, Myburg AA, Grattapaglia D, Vaillancourt RE, Kilian A: A high-density Diversity Arrays Technology (DART) microarray for genome-wide genotyping in Eucalyptus. Plant Methods 2010, 6:16.

47. Sexton TR, Henry RJ, McManus LJ, Bowen S, Shepherd M: Capture of assay template by multiplex PCR of long amplicons for genotyping SNPs and InDels with MALDI-TOF mass spectrometry. Molecular Breeding 2010, 25(3):471-480.

48. Thumma BR, Nolan MR, Evans R, Moran GF: Polymorphisms in cinnamoyl CoA reductase $(C C R)$ are associated with variation in microfibril angle in Eucalyptus spp. Genetics 2005, 171(3):1257-1265.

49. Kulheim C, Yeoh SH, Maintz J, Foley WJ, Moran GF: Comparative SNP diversity among four Eucalyptus species for genes from secondary metabolite biosynthetic pathways. Bmc Genomics 2009, 24(10):452.

50. Matukumalli LK, Lawley CT, Schnabel RD, Taylor JF, Allan MF, Heaton MP, O'Connell J, Moore SS, Smith TPL, Sonstegard TS, et al: Development and Characterization of a High Density SNP Genotyping Assay for Cattle. Plos One 2009, 4(4).

51. Wang S, Sha Z, Sonstegard T, Liu H, Xu P, Somridhivej B, Peatman E, Kucuktas H, Liu Z: Quality assessment parameters for EST-derived SNPS from catfish. BMC genomics 2008, 9(1):450.

52. Akhunov E, Nicolet C, Dvorak J: Single nucleotide polymorphism genotyping in polyploid wheat with the Illumina GoldenGate assay. Theor Appl Genet 2009, 119(3):507-517.

53. Hosmer DW, Lemeshow S: Applied Logistic Regression. New York; Chichester: John Wiley \& Sons, Inc; 22000.

54. Ladiges PY, Udovicic F, Nelson G: Australian biogeographical connections and the phylogeny of large genera in the plant family Myrtaceae. Journal of Biogeography 2003, 30(7):989-998.

55. Crisp M, Cook L, Steane D: Radiation of the Australian flora: what can comparisons of molecular phylogenies across multiple taxa tell us about the evolution of diversity in present-day communities? Philosophical
Transactions of the Royal Society of London Series B-Biological Sciences 2004, 359(1450):1551-1571.

56. Eid J, Fehr A, Gray J, Luong K, Lyle J, Otto G, Peluso P, Rank D, Baybayan P, Bettman $B$, et al: Real-time DNA sequencing from single polymerase molecules. Science 2009, 323(5910):133-138.

57. Grattapaglia D, Resende MDV: Genomic Selection in forest tree breeding Tree Genetics \& Genomes 2010.

58. Grattapaglia D, Alfenas AC, Coelho ASG, Bearzoti E, Pappas GJ, Pasquali G, Pereira G, Colodette J, Gomide JL, Bueno J, et al: Building resources for molecular breeding of Eucalyptus. International IUFRO Conference: Eucalyptus in a changing world: October 11-15 2004; Aveiro Portugal RAIZ, Instituto Investigação da Floresta e Papel, Portugal; 2004, 20-32.

59. Cross_match. [http://www.phrap.org/].

60. Papanicolaou A, Stierli R, Ffrench-Constant R, Heckel D: Next generation transcriptomes for next generation genomes using est2assembly. BMC bioinformatics 2009, 10(1):447.

61. Ewing B, Hillier L, Wendl MC, Green P: Base-calling of automated sequencer traces using phred. I. Accuracy assessment. Genome research 1998, 8(3):175-185

62. Phrap. [http://www.phrap.org/]

63. Zimmermann J, Lipt $\tilde{j} j k$ Z, Hazelhurst S: A Method for Evaluating the Quality of String Dissimilarity Measures and Clustering Algorithms for EST Clustering. Fourth IEEE Symposium on Bioinformatics and Bioengineering: 2004; Taichung, Taiwan, ROC 2004.

64. Hazelhurst S, Hide W, Lipt Ãjk Z, Nogueira R, Starfield R: An overview of the wcd EST clustering tool. Bioinformatics (Oxford, England) 2008, 24(13):1542-1546.

65. Wegrzyn J, Lee J, Liechty J, Neale D: PineSAP-sequence alignment and SNP identification pipeline. Bioinformatics (Oxford, England) 2009, 25(19):2609-2610.

66. Chevreux B, Pfisterer T, Drescher B, Driesel AJ, Muller WEG, Wetter T, Suhai S: Using the miraEST assembler for reliable and automated mRNA transcript assembly and SNP detection in sequenced ESTs. Genome Research 2004, 14(6):1147-1159.

67. Marth GT, Korf I, Yandell MD, Yeh RT, Gu ZJ, Zakeri H, Stitziel NO, Hillier L, Kwok PY, Gish WR: A general approach to single-nucleotide polymorphism discovery. Nature Genetics 1999, 23(4):452-456.

68. Slater GS, Birney E: Automated generation of heuristics for biological sequence comparison. Bmc Bioinformatics 2005, 15(6):31.

69. Goicoechea M, Lacombe E, Legay S, Mihaljevic S, Rech P, Jauneau A, Lapierre C, Pollet B, Verhaegen D, Chaubet-Gigot N, et al: EgMYB2, a new transcriptional activator from Eucalyptus xylem, regulates secondary cell wall formation and lignin biosynthesis. Plant Journal 2005, 43(4):553-567.

70. Legay S, Lacombe E, Goicoechea M, Briere C, Seguin A, Mackay J, GrimaPettenati J: Molecular characterization of EgMYB1, a putative transcriptional repressor of the lignin biosynthetic pathway. Plant Science 2007, 173(5):542-549.

71. Paux E, Carocha V, Marques C, de Sousa AM, Borralho N, Sivadon P, GrimaPettenati J: Transcript profiling of Eucalyptus xylem genes during tension wood formation. New Phytologist 2005, 167(1):89-100.

72. Paux E, Tamasloukht M, Ladouce N, Sivadon P, Grima-Pettenati J: Identification of genes preferentially expressed during wood formation in Eucalyptus. Plant Molecular Biology 2004, 55(2):263-280.

73. Kirst M, Basten CJ, Myburg AA, Zeng ZB, Sederoff RR: Genetic architecture of transcript-level variation in differentiating xylem of a eucalyptus hybrid. Genetics 2005, 169(4):2295-2303.

74. Kirst M, Myburg AA, De Leon JPG, Kirst ME, Scott J, Sederoff R: Coordinated genetic regulation of growth and lignin revealed by quantitative trait locus analysis of cDNA microarray data in an interspecific backcross of Eucalyptus. Plant Physiology 2004, 135(4):2368-2378.

75. Grattapaglia D, Sederoff R: Genetic-Linkage Maps of Eucalyptus-Grandis and Eucalyptus-Urophylla Using a Pseudo-Testcross - Mapping Strategy and Rapd Markers. Genetics 1994, 137(4):1121-1137.

76. Fan JB, Gunderson K, Bibikova M, Yeakley J, Chen J, Wickham Garcia E, Lebruska L, Laurent M, Shen R, Barker D: Illumina universal bead arrays. Methods in enzymology 2006, 410:57-73.

doi:10.1186/1471-2229-11-65

Cite this article as: Grattapaglia et al.: High-throughput SNP genotyping in the highly heterozygous genome of Eucalyptus: assay success, polymorphism and transferability across species. BMC Plant Biology 2011 11:65. 